\title{
Treatment of ischaemic heart disease and stroke in individuals with psychosis under universal healthcare
}

\author{
Stephen Kisely, Leslie Anne Campbell and Yan Wang
}

\section{Background}

Most data on the quality of vascular care for individuals with psychiatric conditions come from countries without universal healthcare.

\section{Aims \\ To investigate the treatment of people with psychosis admitted for ischaemic heart disease or stroke under universal healthcare.}

\section{Method}

A population-based study of administrative data comparing Canadians with and without a history of schizophrenia or related psychosis $(n=65039)$.

\section{Results}

Of 49248 admissions for ischaemic heart disease, 1285 had a history of psychosis. Despite a higher 1-year mortality, they were less likely to receive guideline-consistent treatment: e.g. coronary artery bypass grafting (adjusted odds ratio $(\mathrm{OR})=0.35,95 \% \mathrm{Cl} 0.25-0.48$ ), beta-blockers (adjusted $\mathrm{OR}=0.82,95 \% \mathrm{Cl} 0.71-0.95)$ and statins (adjusted $\mathrm{OR}=0.51$, $95 \% \mathrm{Cl} 0.41-0.63)$. Of 15791 admissions for stroke, 594 had a history of psychosis. Despite higher 1-year mortality rates, they were less likely to receive cerebrovascular arteriography or warfarin.

\section{Conclusions}

People with a history of psychosis do not receive equitable levels of vascular care under universal healthcare.

\section{Declaration of interest}

None.
Although circulatory disease is the major cause of excess death in people with psychiatric disorders, ${ }^{1,2}$ most studies indicate that they experience inequities in specialist medical care. ${ }^{3-6}$ The discrepancy is generally, but not always, greater for severe mental disorders such as schizophrenia, depending on the study population. ${ }^{3-7}$ However, studies have generally been of specific groups in the USA, such as the over-65s, the commercially insured or veterans, and may not apply to jurisdictions where health coverage extends to all residents. ${ }^{3-7}$ Results from countries such as Canada and Australia may therefore be more generalisable. Populationbased studies from both countries have shown that although the risk of mortality is greater for people with psychiatric conditions, they are less likely to receive revascularisation procedures. ${ }^{2,8,9}$ Again, the discrepancy is greatest for severe psychiatric disorder such as schizophrenia and other non-affective psychosis. However, these population-based studies did not compare individuals with psychiatric conditions with controls without such conditions following admission for circulatory disease, or consider differences in the use of vascular drugs such as angiotensin-converting enzyme (ACE) inhibitors, beta-blockers, or statins. In addition, most work has focused on ischaemic heart disease. There is less information on whether individuals with psychiatric conditions receive equitable care for other circulatory disease, such as stroke, but it is already known that socioeconomic status affects the quality of stroke care even under universal health coverage. ${ }^{10}$ Our hypothesis was therefore that psychiatric status would also affect rates of specialised procedures or the prescription of vascular medications, following first admission for either ischaemic heart disease or stroke.

\section{Method}

We compared the quality of care of people with and without severe mental illness after first admission to hospital for ischaemic heart disease or stroke under universal healthcare. By universal healthcare, we mean coverage that is extended to all eligible residents of a jurisdiction. The method of payment varies. In some European countries it is met through insurance, whereas in others such as Canada and Great Britain, it is funded through general taxation and is free at the point of delivery. In a third group of countries, the cost is met through compulsory insurance, with or without co-payment.

We focused on schizophrenia and other non-affective psychoses. For brevity, we will refer to all these disorders as psychosis for the rest of the paper. We studied interventions known to reduce morbidity and mortality. ${ }^{11,12}$ These variables have been suggested as indicators of quality of vascular care following admission for ischaemic heart disease or stroke in Canada. ${ }^{10,13}$ The Capital District Health Authority Research Ethics Board approved the protocol.

\section{Setting}

Nova Scotia is a province on the Atlantic seaboard of Canada with a population of just under one million. Halifax is the major metropolitan centre. Under the Canada Health Act, all Canadian residents are entitled to in-patient or out-patient care that is free at the point of delivery. Individuals receive treatment at publicly funded facilities, or are seen by private specialists or general practitioners who bill the provincial health plan. There are no private hospital beds to date. The linked administrative databases of Nova Scotia therefore cover reimbursed health service use in the public and private sectors across the whole province, including in-patient, out-patient and community physician contacts with both specialist and primary care services. This includes all physicians in private practice.

\section{Data sources}

We used the following administrative databases from the Population Health Research Unit at Dalhousie University from 
1995 to 2001: the Vital Statistics database for mortality; the Discharge Abstract Database for admission type and diagnosis; physician billings for specialists and family physicians in public and private practice, including service date and diagnosis; the Mental Health Outpatient Information System for demographics, diagnoses and visits to mental health clinicians in publicly funded facilities; and the Seniors' Pharmacare database of claims under the province's drug plan for eligible people over 65 years old, including dose and duration (www.phru.dal.ca/about/).

An advantage of the Mental Health Outpatient Information System is that it collects information on contacts with all mental health clinicians, not just physicians. Nova Scotia is one of the few Canadian provinces to capture this type of data. ${ }^{14}$ Seniors' Pharmacare provides coverage for approximately $90 \%$ of the over-65s in the province. Only the most affluent individuals are not covered.

We linked data by using the provincial health card number as a unique identifier. Health card numbers were present in more than $99 \%$ of the records, irrespective of the database, and were encrypted to ensure confidentiality. ${ }^{9}$

Health Canada and the Public Health Agency of Canada have both used administrative data-sets for chronic disease surveillance. ${ }^{14,15}$ Although these data were collected for billing, rather than surveillance, studies using these data-sets for disease surveillance have shown acceptable accuracy over time and against other measures. ${ }^{14-16}$

\section{Sample and methods}

This was a retrospective cohort study. We identified individuals who had an initial admission for each condition of interest during the study period. We used the following ICD-9 $9^{17}$ diagnoses or ICD-10 ${ }^{18}$ equivalents: ischaemic heart disease (410-414) and stroke (431-438). Two separate analyses were conducted for people admitted with ischaemic heart disease and for those admitted with stroke. We then compared rates of 28-day and 1-year fatality, specialised procedures (up to 1-year post-admission), and the prescription of morbidity- and mortalityreducing vascular medications within 90 days of discharge among those with psychosis with those without psychosis.

We derived our sample of individuals with psychosis in the following way. We initially used the definition of the Public Health Agency of Canada for surveillance of treated psychiatric disorders. ${ }^{15}$ Individuals were included if they had at least one visit recorded in the physician billings or mental health out-patient databases, or a discharge from any hospital within 1 year of their initial admission for circulatory disease, with a diagnosis in the most responsible diagnosis field of the following ICD codes: ICD-9 from 290 to 319 inclusive, or their ICD-10 and DSM-IV ${ }^{19}$ equivalents. We used a hierarchy of in-patient $v$. outpatient, and specialist $v$. primary care, reflecting both the increasing proportion of people with severe mental illness and data reliability. It also allowed comparison with Australian data. ${ }^{1,2}$

We included all individuals in contact with psychiatric services within the 12 months preceding their initial admission for circulatory disease. The focus for this paper was on schizophrenia and other non-affective psychoses (295, 297, 298), including schizophreniform, schizoaffective, paranoid and other non-organic psychotic disorders. We will describe all these diagnoses as 'psychosis' for the rest of the paper. We then compared people with psychosis with the rest of the sample, including other psychiatric disorders defined as follows: dementia and other organic conditions (290-294), alcohol/drug disorders (303-305) and all other psychiatric disorders, including mood disorders, neuroses, personality disorders and adjustment reactions (296, 300-302,
306-319). Finally, we also conducted sensitivity analyses of the effect of comparing those with psychosis with the non-psychiatric group only.

For people admitted with ischaemic heart disease we identified right- and left-heart catheterisation, percutaneous transluminal coronary angioplasty with or without stent and coronary artery bypass grafting. ${ }^{11,12}$ For those over 65 years old, we identified prescriptions filled within 90 days of discharge from hospital for the following medications known to reduce morbidity or mortality in randomised controlled trials: beta-blockers, ACE inhibitors, statins, angiotensin receptor blockers and clopidogrel. $^{12,20-25}$ These medications are therefore seen in Canada as indicators of the quality of vascular care. ${ }^{13}$ For individuals admitted with stroke, we identified two procedures: cerebrovascular arteriography and carotid endarterectomy, and for the over-65s, prescriptions for clopidogrel, ticlopidine and warfarin filled within 90 days of discharge. ${ }^{10,26}$ As before, we compared these individuals with psychosis with the rest of the sample, including those with other psychiatric disorders, as well as conducting sensitivity analyses of the effect of comparing individuals with psychosis with the non-psychiatric group only.

Rates of procedures and prescription drug use in the group with psychosis and the group without psychosis were examined for up to 1 year after the index cardiological or stroke admission. Significance was tested using odds ratios and 95\% confidence intervals. We then used logistic regression to examine the effect of psychosis on each outcome (death, specialist procedure or vascular medication) while adjusting for potential confounders. We included psychiatric diagnosis, age, gender, socioeconomic status, comorbid illness, and place of residence (in distance in kilometres from metropolitan Halifax). Income levels of the study population were classified into the quartiles of the 1996 census average household income for the Nova Scotia population by the postal code at the time of initial contact. The presence of medical comorbidity was assessed using the modified CharlsonDeyo index score. This gives a weighted summary score based on 1-year mortality risk. ${ }^{27}$

\section{Results}

\section{Ischaemic heart disease}

A total of 49248 Nova Scotians were admitted with ischaemic heart disease. Their mean age was 65.4 (s.d.=13.6) and 58\% $(n=28347)$ were male. The overall 28 -day and 1 -year mortality rates were 8 and $12 \%$ respectively. Rates at 1 year for cardiac catheterisation were higher $(20 \%)$ than those for percutaneous transluminal coronary angioplasties $(7 \%)$ and coronary artery bypass grafts (9\%), reflecting the fact that cardiac catheterisation is typically the first step in a decision to offer percutaneous transluminal coronary angioplasty or coronary artery bypass grafting. ${ }^{6}$

Of the sample, 11139 (23\%) had had previous contact with primary or secondary care for psychiatric problems and 1285 people $(3 \%)$ had a history of psychosis. Table 1 compares those with psychosis with those without. Individuals with psychosis had significantly higher 28-day and 1-year mortality rates than the rest of the sample, but lower rates of all three specialised procedures (Table 1).

Of other variables that might be associated with the outcomes of interest, people who were older or female and those with comorbid illness had increased mortality rates but a reduced chance of receiving specialised procedures (online Table DS1). By contrast, more affluent individuals had a reduced mortality risk but a greater chance of receiving specialised procedures (online 


\begin{tabular}{|c|c|c|c|c|c|}
\hline & Psychosis, $n$ (\%) & No psychosis, $n$ (\%) & Unadjusted OR (95\% Cl) & Adjusted OR $(95 \% \mathrm{Cl})^{\mathrm{b}}$ & $P$ \\
\hline \multicolumn{6}{|l|}{ Mortality and procedures ${ }^{a}$} \\
\hline 28-day mortality & $153(11.9)$ & $3821(8.0)$ & $1.56(1.32-1.86)$ & - & - \\
\hline 1-year mortality & $243(18.9)$ & $5664(11.8)$ & $1.74(1.51-2.01)$ & $1.27(1.09-1.48)$ & 0.002 \\
\hline Cardiac catheterisation & $112(8.7)$ & $9639(20.1)$ & $0.38(0.31-0.46)$ & $0.47(0.38-0.58)$ & $<0.0001$ \\
\hline PTCA & $31(2.4)$ & $3520(7.3)$ & $0.31(0.22-0.46)$ & $0.41(0.29-0.59)$ & $<0.0001$ \\
\hline Coronary artery bypass grafting & $35(2.7)$ & $4355(9.1)$ & $0.28(0.20-0.39)$ & $0.35(0.25-0.49)$ & $<0.0001$ \\
\hline \multicolumn{6}{|l|}{ Medication $^{c}$} \\
\hline Beta-blockers & $288(32.3)$ & $10261(38.4)$ & $0.76(0.66-0.88)$ & $0.82(0.71-0.95)$ & 0.01 \\
\hline ACE inhibitors & $216(24.2)$ & $7072(26.5)$ & $0.89(0.76-1.04)$ & - & - \\
\hline Statins & $102(11.4)$ & $6063(22.7)$ & $0.44(0.36-0.54)$ & $0.51(0.41-0.63)$ & $<0.0001$ \\
\hline Angiotensin receptor blockers & $37(4.1)$ & $1244(4.7)$ & $0.89(0.63-1.24)$ & - & - \\
\hline Clopidogrel & $28(3.1)$ & $1336(5.0)$ & $0.62(0.42-0.90)$ & - & - \\
\hline
\end{tabular}

Table DS1). Distance in kilometres to Halifax, where specialised services are concentrated, was associated with a reduced chance of receiving specialised procedures (online Table DS1).

We included all the above variables in our logistic regression model (i.e. psychiatric diagnosis, age, gender, socioeconomic status, comorbidity and place of residence). On multivariate analysis, people with psychosis had significantly higher 1-year mortality rates than the rest of the cohort, and lower rates of all three specialised procedures even after adjusting for the other variables (Table 1).

Data from Pharmacare were available on 27629 people who were over 65 years old. Rates of vascular drug prescription ranged from 5 to $38 \%$. Of these, 5118 (18\%) had had previous contact with primary or secondary care for psychiatric problems. Individuals with psychosis $(n=893)(3 \%)$ were significantly less likely to be prescribed beta-blockers and statins compared with the general population and those with other psychiatric diagnoses (Table 1). Older people, and those with comorbid illness, were also less likely to receive vascular medications (online Table DS2). The results for those with psychosis remained significant on multivariate analysis (Table 1).

\section{Stroke}

A total of 15791 Nova Scotians were admitted with stroke. Their mean age was $70.4($ s.d. $=13.1)$ and $51.1 \%(n=8071)$ were male. The overall 28-day and 1-year mortality rates were 5 and $10 \%$ respectively. Six per cent of the sample had received one or other of the procedures.
Of the sample, $3632(23 \%)$ had had previous contact with primary or secondary care for psychiatric problems and 594 people (4\%) had a history of psychosis. Individuals with psychosis had significantly higher 1-year mortality rates than the rest of the cohort but lower rates of both specialised procedures (cerebrovascular arteriography and carotid endarterectomy) (Table 2). As with ischaemic heart disease, female and older people had increased mortality rates but a reduced chance of receiving specialised procedures (online Table DS3). There was a less clear pattern for the other variables (online Table DS3).

On multivariate analysis, individuals with psychosis still had significantly higher 1-year mortality rates than the rest of the cohort but lower rates of cerebrovascular arteriography even after adjusting for the other variables (Table 2).

Data from Pharmacare were available on 11423 people who were over 65 years old. Rates of vascular drug prescription ranged from 3 to $8 \%$. Of these, 2040 (18\%) had had previous contact with primary or secondary care for psychiatric problems. Individuals with psychosis $(n=442)(4 \%)$ were significantly less likely to receive warfarin compared with the general population and those with other psychiatric diagnoses, remaining significant on multivariate analysis (Table 2). Again, older people were also less likely to receive vascular medications (online Table DS4).

\section{Sensitivity analyses}

We found similar results for both ischaemic heart disease and stroke when we compared those with psychosis with the nonpsychiatric group only.

\begin{tabular}{|c|c|c|c|c|c|}
\hline & Psychosis, $n$ (\%) & No psychosis, $n$ (\%) & Unadjusted OR (95\% Cl) & Adjusted OR $(95 \% \mathrm{CI})^{\mathrm{b}}$ & $P$ \\
\hline \multicolumn{6}{|l|}{ Mortality and procedures ${ }^{a}$} \\
\hline 28-day mortality & $37(6.2)$ & $684(4.5)$ & $1.41(1.00-1.98)$ & - & - \\
\hline 1-year mortality & $91(15.3)$ & $1523(10.0)$ & $1.62(1.29-2.04)$ & $1.49(1.17-1.88)$ & 0.001 \\
\hline Cerebrovascular arteriography & $9(1.5)$ & $497(3.3)$ & $0.46(0.23-0.88)$ & $0.47(0.24-0.93)$ & 0.03 \\
\hline Carotid endarterectomy & $11(1.9)$ & $441(2.9)$ & $0.63(0.34-1.16)$ & - & - \\
\hline \multicolumn{6}{|l|}{ Medication $^{c}$} \\
\hline Ticlopidine & $15(3.4)$ & 387 (3.5) & $0.96(0.57-1.63)$ & - & - \\
\hline Warfarin & $22(5.0)$ & $945(8.6)$ & $0.56(0.36-0.86)$ & $0.55(0.36-0.85)$ & 0.01 \\
\hline Clopidogrel & $11(2.5)$ & $338(3.1)$ & $0.80(0.44-1.48)$ & - & - \\
\hline
\end{tabular}




\section{Discussion}

\section{Comparisons with previous studies}

People with histories of psychiatric contact have more than double the mortality of the general population, the major causes being ischaemic heart and cerebrovascular disease rather than suicide. ${ }^{1,2}$ Possible explanations include differences in incidence, lifestyle, use of psychotropic medication or inequitable access to appropriate healthcare. Studies from the USA have highlighted how people with psychiatric disorders are less likely to receive appropriate secondary care and that, in some cases, these deficits seemed to explain much of the subsequent excess mortality. ${ }^{3-6}$ However, studies were restricted to specific groups defined by age, database or health coverage..$^{3-7}$ In the case of studies of the over-65s, samples were restricted to mental disorders recorded as a secondary diagnosis on admission with ischaemic heart disease and so may be an underestimate given the low reported prevalence of psychiatric morbidity (5\%). ${ }^{3,4}$ A study of individuals covered by the Healthcare Investment Analysts (HCIA) Sachs Projected Inpatient database was limited by the same definition of psychiatric morbidity. ${ }^{5}$ Findings for the commercially insured may also be less applicable to people with severe mental illness as they are less likely to have private insurance. ${ }^{1,6}$ The study where there was the least difference in the care of people with psychosis and those without was of veterans and this system most resembles care in countries such as Great Britain and Canada. ${ }^{7}$ Providers are salaried employees, out-of-pocket costs for patients are low and coverage or eligibility are not tied to employment. However, this sample is still subject to selection bias in that benefits are linked to past military service, and only those with disability related to military service are eligible for the full range of services. Findings from the USA results may therefore be less relevant to jurisdictions with universal healthcare.

\section{Quality of care under a universal healthcare system}

Our results suggest that people with psychosis admitted for circulatory disease experience similar disadvantages under universal healthcare to those in the $\mathrm{USA}^{3-6}$ following admission for circulatory disease. They have the highest mortality but least chance of receiving many specialised interventions or circulatory medications. The results are also consistent with findings from ambulatory and primary care where people with severe mental illness were less likely to be assessed or treated for hyperlipidaemia. ${ }^{28-32}$ Finally, this research extends previous findings for ischaemic heart disease by including stroke, with the finding of similar disparities in the quality of care.

\section{The role of socioeconomic status}

In common with other Canadian studies of cancer and stroke care, we found that socioeconomic status had an effect on access to procedures and outcomes. ${ }^{10,33}$ Under Canada's universal healthcare system, the ability to pay or private health insurance coverage should not influence access to healthcare. Even so, wealthier communities may be more attractive locations for specialist physicians, leading to a concentration of specialised resources. ${ }^{10}$ Conversely, individuals with lower incomes may have greater problems in navigating the health system, difficulties in communication or have other needs competing with healthcare. ${ }^{10,33}$ Socioeconomic status may be related to other factors, such as education level, which also influence access. ${ }^{33}$ Some of these explanations may equally apply to other marginalised groups such as people with psychosis.

\section{Study implications}

It is commonly assumed that lifestyle (e.g. obesity, alcohol or tobacco use) explains the increased mortality of individuals with psychosis. However, studies of cancer, where many of the risk factors are the same as for circulatory disease (e.g. cigarettes, alcohol or diet), suggest that lifestyle is unlikely to be the sole explanation. ${ }^{34-36}$ Other patient-based explanations could include lower adherence to treatment or an inability to give informed consent. ${ }^{37}$ People with psychiatric disorders may also have more general difficulties with access to healthcare shared by other marginalised groups as discussed above.

It is also possible that physicians are reluctant to offer some procedures because of the ensuing psychological stress, concerns about capacity or cooperation with post-operative care, or the presence of contraindications such as smoking. ${ }^{38}$ In addition, individuals with psychiatric disorders may be more at risk of developing complications following medical or surgical interventions ${ }^{39}$ or of having poorer outcomes post-operatively. ${ }^{40}$

These explanations are less relevant to the prescription of medications known to reduce subsequent morbidity and mortality. For instance, contraindications to specialised interventions such as smoking or problems with informed consent apply less to the prescription of vascular drugs such as ACE inhibitors, beta-blockers, or statins. The concern for providers of healthcare, therefore, is that appropriate treatments are not offered because of the stigma of mental disorders in general medical settings. ${ }^{32,41}$

Our findings are particularly relevant given the increasing rates of metabolic syndrome in people with psychosis. Individuals with schizophrenia are at increased risk of metabolic syndrome disorders at rates of between one and a half and five times that of the general population. ${ }^{42,43}$ In turn, people with metabolic syndrome are two to three times as likely to have a heart attack or stroke as those without. ${ }^{42,43}$ Although primary prevention is the approach of choice, ${ }^{43}$ it is likely that greater numbers of people with psychosis will experience complications of metabolic syndrome such as ischaemic heart disease and stroke. Ensuring equitable access to interventions for these disorders will be of growing importance. Aside from improved screening and monitoring, better coordination between general practitioners, psychiatrists and physicians may address the underuse of interventions of proven benefit.

The rigid application of guidelines, such as smoking as a contraindication to certain procedures, may also disadvantage people with psychosis. The importance of ensuring access to appropriate treatment is underlined by results from the USA, which suggest that quality of medical care seems to explain a substantial portion of the excess mortality experienced by individuals with schizophrenia. ${ }^{4}$

\section{Study limitations}

We used routinely collected administrative data that may be subject to recording bias. Data on access to prescriptions were limited to individuals eligible for Pharmacare benefits over the age of 65 years old. The most affluent $10 \%$ of the over-65s in the province are not covered. However, given that our focus is on people with psychoses, we are unlikely to have not captured most of the relevant individuals in this age group. We did not have data on marital status, length of time since first contact with services and educational status, all of which may influence mortality. Administrative data do not contain indicators of circulatory disease severity, such as level of consciousness and functional status. Neither were we able to study the effect of lifestyle or behavioural issues such as alcohol, drug or tobacco use. However, when assessing the risk of ischaemic heart disease, 
unmeasured risk factors result in little bias, and the effect is to reduce differences between groups. ${ }^{44}$ We were also unable to consider the effect of legal status. However, two studies from Australia and the USA showed no difference in all-cause mortality rates between involuntary and voluntary patients. ${ }^{1,45}$

Overall rates of specialised procedures and circulatory disease prescription were lower in the Nova Scotian administrative data than have been reported from elsewhere in Canada or the United States. ${ }^{6,12}$ In the case of Pharmacare data, this may reflect lower prescription rates in the over-65s compared with younger people. Another explanation might be different denominators. Some studies have focused on individuals in whom interventions were particularly indicated. We were not able to stratify our sample by groups with and without specific indications for a given intervention and this could have biased our results if these individuals were not evenly distributed between the groups with psychosis and those without. On the other hand, it is unlikely to explain every one of our results. Furthermore, as noted previously, unmeasured risk factors result in little bias and tend to reduce differences between comparison groups. ${ }^{44}$ In addition, we were able to adjust for other important clinical predictors, including age, gender, socioeconomic status and comorbid illness. Our data also cannot determine the contribution of system-, physician- or patient-based factors to the observed gaps in treatment. Finally, Pharmacare does not cover over-the-counter medications such as aspirin. We therefore only have information on clopidogrel for this class of medication.

\section{Next steps}

Our findings may have implications for other countries with universal healthcare such as Great Britain. Further research could help establish the relative contribution of system-, physician- or patient-based factors and how these can be addressed. For some individuals, this could be because of difficulties in communication, registering with a family physician or scheduling appointments because of frequent changes of address. Service providers should also be prepared to accommodate the needs of people with mental illness, as they do those with physical difficulties, to enhance access to medical care.

\footnotetext{
Stephen Kisely, MD, MSC, University of Queensland, Brisbane, Australia and Dalhousie University, Halifax, Nova Scotia, Canada; Leslie Anne Campbell, BSCN, MSc, Health Outcomes Research Unit, Capital District Health Authority, Halifax, Nova MSC, Health Outcomes Research Unit, Capital District Health Authority, Halifax,
Scotia, Canada; Yan Wang, MCS, Population Health Research Unit, Dalhousie University, Halifax, Nova Scotia, Canada

Correspondence: Stephen Kisely, Room 518(A), McGregor Building (No 64), Queensland Centre for Health Data Services, The University of Queensland, St Lucia, Brisbane, Queensland 4072, Australia. Email: s.kisely@uq.edu.au

First received 7 Apr 2009, final revision 8 Jul 2009, accepted 27 Jul 2009
}

\section{Funding}

This study was supported by a Grant-in-Aid award (2004-05) from the Heart and Stroke Foundation of Nova Scotia.

\section{Acknowledgements}

The data used in this report were made available by the Population Health Research Unit (PHRU) within Dalhousie University's Department of Community Health and Epidemiology. The PHRU is a university-based research and support group conducting systematic research into population health, health services and their interrelationships. The Province of Nova Scotia supplies PHRU with complete Medicare and hospital files suitable for research purposes. The Unit also has access to a variety of other data sources, including clinical databases and large-scale population surveys. Although this research is based on data obtained from the PHRU the observations and opinions expressed are those of the data obtained from the PHRU the observations and opinions expressed are those of the
authors and do not represent those of the PHRU. The Capital District Health Authority Research Ethics Board approved the protocol.

\section{References}

1 Lawrence DM, Jablensky AV, Holman CDJ, Pinder TJ. Mortality in Western Australian psychiatric patients. Soc Psychiatry Psychiatr Epidemiol 2000; 35: 341-7.

2 Lawrence DM, Holman CDJ, Jablensky AV, Hobbs MST. Death rate from ischaemic heart disease in Western Australian psychiatric patients 1980-1998. Br J Psychiatry 2003; 182: 31-6.

3 Druss BG, Bradford DW, Rosenheck RA, Radford MJ, Krumholz HM. Mental disorders and use of cardiovascular procedures after myocardial infarction. JAMA 2000; 283: 506-11.

4 Druss BG, Bradford D, Rosenheck RA, Radford MJ, Krumholz HM. Quality of medical care and excess mortality in older patients with mental disorders. Arch Gen Psychiatry. 2001; 58: 565-72.

5 Young JK, Foster DA. Cardiovascular procedures in patients with mental disorders. JAMA 2000; 283: 3198.

6 Jones LE, Carney CP. Mental disorders and revascularization procedures in a commercially insured sample. Psychosom Med 2005; 67: 568-76.

7 Petersen LA, Normand SL, Druss BG, Rosenheck RA. Process of care and outcome after acute myocardial infarction for patients with mental illness in the VA health care system: are there disparities? Health Serv Res 2003; 38: 41-63.

8 Lawrence D, Jablensky AV, Holman CD. Preventable Physical IIIness in People with Mental IIIness. University of Western Australia, 2001.

9 Kisely S, Cox M, Smith M, Lawrence D, Maaten S. Does inequitable access to cardiological or neurological procedures contribute to preventable mortality in people with mental illness? Can Med Assoc Journal 2007; 176: 779-84.

10 Kapral MK, Wang H, Mamdani M, Tu JV. Effect of socioeconomic status on treatment and mortality after stroke. Stroke 2002; 33: 268-73.

11 Eagle, KA, Guyton GA, Davidoff R, Edwards FH, Ewy GA, Gardner TJ, et al. ACC/AHA 2004 guideline update for coronary artery bypass graft surgery: a report on Practice Guidelines (Committee to Update the 1999 Guidelines for Coronary Artery Bypass Graft Surgery). Circulation 2004; 110: 1168-76.

12 Department of Health. National Service Framework for Coronary Heart Disease. Department of Health, 2000 (http://www.dh.gov.uk/en/ Publicationsandstatistics/Publications/PublicationsPolicyAndGuidance/ DH_4094275).

13 Tran CTT, Lee DS, Flintoft VF, Higginson L, Grant FC, Tu JV, et al. CCORT/CCS quality indicators for acute myocardial infarction care. Can J Cardiol 2003; 19: 38-45.

14 National Diabetes Surveillance System. Responding to the Challenge of Diabetes in Canada. First Report of the National Diabetes Surveillance System. Health Canada, 2003 (http://www.phac-aspc.gc.ca/ccdpc-cpcmc/ ndss-snsd/english/pubs_reports/pdf/WEB_NDSS_English_Report-nocover.pdf).

15 Kisely S, Lin E, Lesage A, Gilbert C, Smith M, Campbell LA, et al. Use of administrative data for the surveillance of mental disorders in 5 provinces. Can J Psychiatry 2009; 54: 571-5.

16 Williams Jl, Young W. Inventory of Studies on the Accuracy of Canadian Health Administrative Databases. Institute for Clinical Evaluative Sciences in Ontario, 1996.

17 World Health Organization. International Statistical Classification of Diseases and Related Health Problems, Ninth Revision (ICD-9). WHO, 1978.

18 World Health Organization. International Statistical Classification of Diseases and Related Health Problems, Tenth Revision (ICD-10). WHO, 1992.

19 American Psychiatric Association. Diagnostic and Statistical Manual of Mental Disorder (4th edn) (DSM-IV). APA, 1994.

20 Yusuf S, Peto R, Lewis J, Collins R, Sleight P. Beta blockade during and after myocardial infarction: an overview of the randomized trials. Prog Cardiovasc Dis 1985; 27: 335-71.

21 Fremantle N, Cleland J, Young P, Mason J, Harrison J. Beta blockade after myocardial infarction: systematic review and meta regression analysis. BMJ 1999; 318: 1730-7.

22 Pfeffer MA, Braunwald E, Moye LA, Basta L, Brown EJ Jr, Cuddy TE, et al. Effect of captopril on mortality and morbidity in patients with left ventricular dysfunction after myocardial infarction: results of the Survival and Ventricular Enlargement Trial. N Engl J Med 1992; 327: 669-77.

23 Yusuf S, Sleight P, Pogue J, Bosch R, Davies R, Dagenais G. Effects of an angiotensin-converting-enzyme inhibitor, ramipril, on cardiovascular events in high-risk patients. N Engl J Med. 2000; 342: 145-53.

24 Reboldi G, Angeli F, Cavallini C, Gentile G, Mancia G, Verdecchia P. Comparison between angiotensin-converting enzyme inhibitors and angiotensin receptor blockers on the risk of myocardial infarction, stroke and death: a meta-analysis. J Hypertens 2008; 26: 1282-9. 
25 Antiplatelet Trialists' Collaboration. Collaborative overview of randomised trials of antiplatelet therapy. Prevention of death, myocardial infarction, and stroke by prolonged antiplatelet therapy in various categories of patients. BMJ 1994; 308: 81-106.

26 Lip GY, Edwards SJ. Stroke prevention with aspirin, warfarin and ximelagatran in patients with non-valvular atrial fibrillation: a systematic review and meta-analysis. Thromb Res 2006; 118: 321-33.

27 Deyo RA, Cherkin DC, Ciol MA. Adapting a clinical comorbidity index for use with ICD-9-CM administrative databases. J Clin Epidemiol 1992; 45: 613-9

28 Bradford DW, Kim MM, Braxton LE, Marx CE, Butterfield M, Elbogen EB. Access to medical care among persons with psychotic and major affective disorders. Psychiatr Serv 2008; 59: 847-52.

29 Kilbourne AM, Welsh D, McCarthy JF, Post EP, Blow FC. Quality of care for cardiovascular disease-related conditions in patients with and without mental disorders. J Gen Intern Med 2008; 23: 1628-33.

30 Hippisley-Cox J, Parker C, Coupland C, Vinogradova Y. Inequalities in the primary care of patients with coronary heart disease and serious mental health problems: a cross-sectional study. Heart 2007; 93: 1256-62.

31 Roberts L, Roalfe A, Wilson S, Lester $\mathrm{H}$. Physical health care of patients with schizophrenia in primary care: a comparative study. Fam Pract 2007; 24: 34-40.

32 Weiss AP, Henderson DC, Weilburg JB, Goff DC, Meigs JB, Cagliero E, et al. Treatment of cardiac risk factors among patients with schizophrenia and diabetes. Psychiatr Serv 2006; 57: 1145-52.

33 Paszat LF, Mackillop WJ, Groome PA, Zhang-Salomons J, Schulze K, Holowaty E. Radiotherapy for breast cancer in Ontario: rate variation associated with region, age and income. Clin Invest Med 1998; 21: 125-34.

34 Carney CP, Woolson RF, Jones L, Noyes R Jr, Doebbeling BN. Occurrence of cancer among people with mental health claims in an insured population. Psychosom Med. 2004; 66: 735-43.
35 Lawrence D, Jablensky AV, Holman CD, Threlfall TJ, Fuller SA. Excess cancer mortality in Western Australian psychiatric patients due to higher case fatality rates. Acta Psychiatrica Scand 2000; 101: 382-8.

36 Kisely S, Sadek J, MacKenzie A, Lawrence D, Campbell LA. Excess cancer mortality in psychiatric patients. Can J Psychiatry 2008; 53: 753-61

37 Rieckmann N, Kronish IM, Haas D, Gerin W, Chaplin WF, Burg MM, et al. Persistent depressive symptoms lower aspirin adherence after acute coronary syndromes. Am Heart J 2006; 152: 922-7.

38 Shander D. Cardiovascular procedures in patients with mental disorders. JAMA 2000; 283: 3198-9.

39 Daumit GL, Pronovost PJ, Anthony CB, Guallar E, Steinwachs DM, Ford DE. Adverse events during medical and surgical hospitalizations for persons with schizophrenia. Arch Gen Psychiatry 2006; 63: 267-72.

40 Mallik S, Krumholz HM, Lin ZQ, Kasl SV, Mattera JA, Roumains SA, et al. Patients with depressive symptoms have lower health status benefits after coronary artery bypass surgery. Circulation 2005; 111: 271-7.

41 Penn DL, Martin J. The stigma of severe mental illness: some potential solutions for a recalcitrant problem. Psychiatric Q 1998; 69: 235-47.

42 Waterreus AJ, Laugharne JD. Screening for the metabolic syndrome in patients receiving antipsychotic treatment: a proposed algorithm. Med J Aust 2009; 190: 185-9.

43 Lambert TJ, Newcomer JW. Are the cardiometabolic complications of schizophrenia still neglected? Barriers to care. Med J Aust. 2009; 190 S39-42.

44 Lévesque LE, Brophy JM, Zhang B. The risk for myocardial infarction with cyclooxygenase-2 inhibitors: a population study of elderly adults. Ann Intern Med 2005; 142: 481-9.

45 Crisanti AS, Love EJ. Mortality among involuntarily admitted psychiatric patients: a survival analysis. SOC Psychiatry Psychiatr Epidemiol. 1999; 34: $627-33$

\section{Psychiatry in the} Old Testament

\section{Did the author of Psalm 30 have cyclothymia or bipolar disorder?}

\section{George Stein}

Psalm 30 is a short psalm used as a song for the dedication of the Temple. It contains a description in religious terminology of two abrupt changes of mood which seem to resemble the mood switches of affective disorder. Examples quoted here are verses 30:3-5 and $30: 8-12$.

30:3 'O Lord, you have brought up my soul from Sheol, restored me to life from among those gone down to the Pit. 4 Sing praises to the Lord, 0 you his faithful ones, and give thanks to his holy name. 5 For his anger is but for a moment: his favour is for a lifetime. Weeping may linger for the night, but joy comes with morning.'

A state of misery is described in verse three (and also in one and two, not quoted here), but an abrupt change of mood seems to occur in the night. The psalm continues:

8 'To you Lord, I cried, and to the Lord I made supplication: 9 What profit is there in my death, if I go down to the Pit? Will the dust praise you? Will it tell of your faithfulness? 10 Hear O Lord and be gracious to me O Lord, be my helper! 11 You have turned my mourning into dancing: you have taken off my sackcloth and clothed me with joy, 12 so that my soul may praise you and not be silent. O Lord my God I will give thanks to you for ever.'

Only a writer who has had personal experience of such an abrupt change of mood, from a state of mourning (depression) to one of joy (elation), could describe it so well. He attributes his improved mood to God whom he thanks. Today, in the clinic, many individuals with affective disorder will report such abrupt mood switches. Those with bipolar I disorder are often too lacking in insight to notice their moods have changed, but people with bipolar II disorder or cyclothymia are all too aware that their mood has improved. Perhaps this author had one of those disorders. 